Memorias del VII Encuentro Nacional de Experiencias en la Enseñanza de la Biología y la Educación Ambiental y II Congreso Nacional de Investigación en la Enseñanza de La Biología

\title{
LAS PRÁCTICAS DE CAMPO PARA LA ENSEÑANZA DE LA PALEOBIOLOGIA Y SU APORTE AL RECONOCIMIENTO DEL PATRIMONIO CULTURAL Y NATURAL EN EDUCACIÓN SECUNDARIA DEL MUNICIPIO FLORESTA, BOYACÁ
}
THE PRACTICES FOR TEACHING AND ITS CONTRIBUTION PALEOBIOLOGY RECOGNITION OF CULTURAL AND NATURAL SECONDARY FOREST TOWNSHIP, BOYACÁ

\section{Paola Sánchez Herrera ${ }^{1}$ Gloria Escobar ${ }^{2}$}

\section{Resumen}

Para la presente ponencia se realiza una descripción de la práctica pedagógica desarrollada al interior del grupo de investigación Conocimiento Profesional del Profesor de Ciencias (CPPC) de la Universidad Pedagógica Nacional, Bogotá (Colombia), en el Municipio de Floresta, Boyacá, en la cual, se busco formular Prácticas de campo para la enseñanza de la Paleobiología que impulsaran al reconocimiento del patrimonio cultural y natural en los profesores y estudiantes de la Institución Héctor Julio Rangel Quintero, donde se realizó la practica integral, que por medio de la indagación de concepciones por parte de las profesoras y el análisis de las mismas, se llegara al reconocimiento de la importancia de las Practicas de campo como estrategia didáctica en la enseñanza de la Biología. La investigación se desarrolló bajo el paradigma cualitativo-interpretativo el cual habla de las asociaciones fundamentales con la investigación o perspectiva cualitativa en donde metodológicamente se caracteriza por el énfasis que se hace en la aplicación de las técnicas de descripción, clasificación y explicación. De acuerdo a lo encontrado en la investigación se concluyó que las profesoras no tienen claro qué es una estrategia didáctica y por ende la falencia y nula realización de éstas en la clase de Biología es un limitante en la enseñanza y aprendizaje de ésta, ya que los estudiantes están acostumbrados a las mismas dinámicas y muchos no se interesan por la Biología, es decir que pierden interés al no tener actividades que los motiven e interesen por conocer la materia en cuestión.

Palabras clave: Prácticas de campo, Paleobiología, enseñanza, patrimonio, Conocimiento Profesional del Profesor de Ciencias.

\footnotetext{
1 Universidad Pedagógica Nacional. Estudiante de IX semestre de Licenciatura en Biología. neop 458@hotmail.com

${ }^{2}$ Universidad Pedagógica Nacional. Profesora del Departamento de Biología araneida542@yahoo.com.mx
} 
Memorias del VII Encuentro Nacional de Experiencias en la Enseñanza de la Biología y la Educación Ambiental y II Congreso Nacional de Investigación en la Enseñanza de la Biología

\section{Abstract}

For the present paper is a description of pedagogical practice developed within the research group of Professor Professional Knowledge Science (CPPC) National Pedagogical University, Bogota (Colombia), in the municipality of Floresta, Boyacá, in which, is looking for field practices make teaching Paleobiology that would prompt a recognition of the cultural and natural heritage in the teachers and students of the institution Hector Julio Rangel Quintero, where the practice was integral to the inquiry through conceptions by the teachers and analyzing them, it came to recognizing the importance of field practices as a teaching strategy in teaching biology. The research was conducted under the qualitative-interpretive paradigm which speaks of fundamental associations with qualitative research or where methodological perspective is characterized by the emphasis placed on the application of the techniques of description, classification and explanation. According to what was found in investigation concluded that teachers are not sure what is a teaching strategy and therefore the failure and no realization of these in Biology class is a constraint on the teaching and learning of this, since the students are used to the same dynamics and many are not interested in biology, ie lose interest by not having activities that motivate and interest in learning about the subject matter.

Keywords: Field Practices, Paleobiology, education, heritage, Knowledge Science Teacher Professional.

\section{Introducción}

La Universidad Pedagógica Nacional, se ha caracterizado por su aporte al desarrollo de la educación nacional, mediante la formación de profesionales para los diferentes niveles del sistema educativo, la investigación, producción y divulgación de pensamiento pedagógico. En consecuencia el Proyecto Curricular de la Licenciatura en Biología (PCLB) está encaminado a la formación de educadores integrales, con un sentido social y político para contribuir con la cultura por medio de investigaciones pedagógicas como las prácticas pedagógicas que aporten a la construcción de un conocimiento pedagógico y biológico centrado en la excelencia en la formación.

Es por esto, que se formulan practicas pedagógicas dentro del DBI como la presente en donde, se proponen las prácticas de campo como una estrategia didáctica con la cual cuentan los profesores de ciencias naturales, para acercar el conocimiento científico a la escuela, además de generar experiencias las cuales permiten desarrollar habilidades cognitivas tanto en profesores como en estudiantes; sin embargo el análisis de estas prácticas de enseñanza de la Biología son escasas, generalmente se han abordado desde la Geología y la 
Memorias del VII Encuentro Nacional de Experiencias en la Enseñanza de la Biología y la Educación Ambiental y II Congreso Nacional de Investigación en la Enseñanza de la Biología

Geografía (Amórtegui, 2011). En el caso de la Paleobiología, se han encontrado caracterizaciones como la de Gil (2006), la cual describe el procedimiento para la colecta de fósiles y su preservación, también se encuentra a Campos (2009), que argumenta la importancia de salidas de campo de corte Paleobiológico para maestros en formación.

Desde la perspectiva del Conocimiento Profesional del Profesor, estas prácticas de campo, son un componente importante dentro del Conocimiento Didáctico del Contenido el cual, es el conocimiento que se necesita para poder transformar los contenidos disciplinares, en contenidos de enseñanza (Valbuena, 2007), en esta medida este conocimiento se encarga de acercar el conocimiento disciplinar a la escuela para que estos contenidos sean enseñables y aprendibles.

Las prácticas de campo en este contexto, deben representar en los profesores, una infinidad de posibilidades en el campo pedagógico y didáctico, las cuales no solamente se centren en la enseñanza de un contenido biológico que debe estar constituido por elementos sustantivos y sintácticos que validan el dominio que el profesor maneja sobre el conocimiento (Valbuena, 2007), sino además de poder lograr un verdadero reconocimiento del valor de lo natural y cultural que se posee en el Municipio, que se logra con una integración de conocimientos, permitiendo el desarrollo de concepciones que lleven a los profesores a generar diferentes posibilidades dentro de su conocimiento didáctico, profesional y contextual para lograr primero la dignificación del maestro rural, segundo poder enseñar en contexto sin limitantes a la hora de implementar estrategias didácticas y tercero ser generador de propuestas que ayuden a la comunidad a mantener y cuidar el patrimonio natural y cultural.

La pregunta problema que guío la propuesta de práctica pedagógica es ¿Cómo la formulación de prácticas de campo posibilita la enseñanza de la Paleobiología e impulsan al reconocimiento del patrimonio cultural y natural en los profesores y estudiantes del municipio de Floresta Boyacá?

De acuerdo con lo anterior, lo que se pretende hacer con la práctica pedagógica, es caracterizar y analizar el papel de las salidas de campo que tienen como tema principal la enseñanza de los fósiles y su aporte para el reconocimiento del patrimonio cultural del Municipio de Floresta, Boyacá, además cómo este ejercicio aporta en la construcción del conocimiento profesional del profesor y en particular cómo fortalecer su conocimiento didáctico por medio de estas prácticas.

\section{Metodología}

La práctica pedagógica integral se realizó en el Municipio de Floresta, Boyacá, en la Institución Educativa Héctor Julio Rangel Quintero, con los estudiantes de Noveno grado, bajo un enfoque investigativo el cual posee unas características 
Memorias del VII Encuentro Nacional de Experiencias en la Enseñanza de la Biología y la Educación Ambiental y II Congreso Nacional de Investigación en la Enseñanza de la Biología

como son la sistematización, la descripción, la explicación, la comparación y la experimentación, está relacionado con los métodos de investigación (como son el deductivo, inductivo, hipotético-deductivo, histórico- comparativo.) éstos, están ligados a las problemáticas del conocimiento y los caminos para llegar a sus posibles soluciones (Cerda, 2001); el método con el cual la investigación se va a guiar es con el Método Inductivo que hace posible el paso de los hechos singulares, a los principios generales (Cerda, 2001). En donde las particularidades de las prácticas de campo ayudarán, además de consolidar a éstas como un campo de conocimiento dentro de la enseñanza de la Biología, también como una estrategia didáctica que posibilita en cualquier entorno el reconocimiento tanto natural como cultural y su valor para la comunidad.

Por estas razones, como ya se había mencionado, la perspectiva que va a tomo la investigación fue de carácter cualitativo, en donde el conocimiento es un producto social y su proceso de producción colectivo, está atravesado por valores, percepciones y significados de los sujetos que lo construyeron. (Galeano, 2009).

Los instrumentos de recolección de datos dentro de la investigación se utilizaron para acceder a la información que se necesitaba para la solución de la pregunta problema; teniendo en cuenta que la investigación va a girar dentro de una perspectiva cualitativa, los instrumentos que se escogieron fueron la observación y la entrevista. El método utilizado para el análisis de la información fue por análisis del contenido.

La investigación se dividió en 3 fases principales que fueron:

- Indagación

- Construcción de las Prácticas de campo

- Modificación y documento de compilación de las Prácticas de campo

\section{Resultados y Discusión}

Desde los resultados y análisis encontrados a partir de la implementación de la entrevista a dos profesoras encargadas del área de Ciencias Naturales en la Institución Educativa Héctor Julio Rangel Quintero, como principal instrumento dentro de la investigación, se generan diferentes planteamientos importantes que merecen tener una discusión en torno al CPPC y los conocimientos que de allí se desprenden a partir del objeto de estudio que en este caso son las prácticas de campo, por medio de las preguntas planteadas en la entrevista.

Las preguntas 1¿Qué estrategias didácticas utiliza para enseñar Ciencias Naturales?, 2 Además del salón, ¿Qué otro espacio utiliza para enseñar Ciencias 
Memorias del VII Encuentro Nacional de Experiencias en la Enseñanza de la Biología y la Educación Ambiental y II Congreso Nacional de Investigación en la Enseñanza de la Biología

Naturales?, 3 ¿Utiliza elementos, recursos o lugares del entorno a la institución para enseñar Ciencias Naturales?, 8, Si enseña acerca del estudio de los fósiles, ¿Qué estrategias utiliza para la enseñanza de este tema?, 9 ¿Realiza Prácticas de campo con sus estudiantes a los yacimientos que se encuentran en el municipio o cerca de él? y 10 ¿Usted cree pertinentes las Prácticas de campo como estrategia en la enseñanza de las Ciencias Naturales? responden al Conocimiento Didáctico del Contenido que como se entiende es el que "se refiere al conocimiento que se necesita para poder transformar los contenidos disciplinares, con el fin de hacerlos más compresibles a los alumnos y facilitar así su aprendizaje. Se trata entonces de hacer de los contenidos disciplinares, contenidos "enseñables y aprendibles", hace referencia a la enseñanza de una disciplina determinada". (Valbuena, 2007:139). En este sentido las profesoras no concuerdan en estas preguntas con lo que responden, ya que las observaciones que se realizaron de las clases no se interpretaba lo que ellas evidencian en la entrevista; si bien, ellas realizan talleres, se apoyan en ayudas audiovisuales y demás, no tienen claro que son estrategias didácticas ya que lo que ellas utilizan son herramientas que les ayudan a llevar el conocimiento disciplinar a la clase, pero estas herramientas no cumplen la labor de hacer más comprensibles ni enseñables el conocimiento, es una simple reproducción y transcripción de los libros de texto o de lecturas que se encuentran en los talleres.

Dentro del CDC, como se ha venido hablando, se encuentran las prácticas de campo, la cual es una estrategia didáctica que según Baldaia (2006) argumenta que "las salidas de campo son indispensables para superar la visión parcial de la realidad que nos proporcionan las actividades de laboratorio o aula y son insustituibles en la comprensión del medio natural" (Baldaia, 2006:34). Partiendo de esto en las preguntas 3,9 y 10, que están referidas a las prácticas de campo, se observa que la falta de construcción de esta estrategia es una falencia que empieza desde los entes administrativos al generar dificultades para la implementación de estas, y por parte de las profesoras por desaprovechar esta estrategia en un contexto tan rico como lo es el Municipio de Floresta en cuanto a la enseñanza de la Paleobiología y la Biología en general.

Las preguntas 4 ¿Conoce usted acerca del patrimonio natural del municipio de Floresta (Boyacá)?, 5, Usted como profesor, al momento de enseñar Ciencias Naturales, ¿Tiene en cuenta el patrimonio que posee el Municipio?, y 6, En clases de Ciencias Naturales, ¿Usted cómo relaciona lo que enseña con el patrimonio que posee el Municipio?, corresponden al Conocimiento Contextual que Según Barnett y Hodson (2001) es el "que determina las características del conocimiento profesional del profesor de ciencias que afectan su quehacer diario y está delimitado por aspectos sociales, culturales, políticos y factores institucionales que afectan el desarrollo de su profesión. Además se habla que los profesores 
Memorias del VII Encuentro Nacional de Experiencias en la Enseñanza de la Biología y la Educación Ambiental y II Congreso Nacional de Investigación en la Enseñanza de la Biología

generan un conocimiento colectivo a partir de las vivencias en el aula y las experiencias que genera el mismo contexto". Con esto se puede determinar que las profesoras no relacionan el contexto con su práctica pedagógica, ya que los temas que ellas imparten en el tema de evolución son totalmente descontextualizados, además desde el currículo empieza el problema de no tener en cuenta el contexto, ya que no se menciona ni se relaciona ningún concepto ni temática dentro de él con el patrimonio natural ni la importancia del mismo.

La pregunta 7 ¿Ha trabajado alrededor del estudio de los Fósiles, o temas relacionados con esta área de estudio? tiene relación con el conocimiento disciplinar que poseen los profesores en el cual se determina las teorías científicas, los postulados y en general el manejo de la disciplina que se está enseñando, encontrando así que las profesoras no poseen conocimiento acerca de los fósiles ni su relación con el contexto en donde están, además en las observaciones de clase se pudo evidenciar que el tema de Evolución lo evitan o simplemente dan a conocer la Escala Geológica del tiempo, ya que "parece ser" las creencias religiosas que ellas poseen son muy arraigadas, igual que la de los estudiantes así que este tipo de temáticas son vistas muy someramente y con ningún análisis.

\section{Conclusiones}

A partir del proceso investigativo es preciso concluir que:

- Las profesoras no tienen claro qué es una estrategia didáctica y por ende la falencia y nula realización de éstas en la clase de Biología es un limitante en la enseñanza y aprendizaje de ésta, ya que los estudiantes están acostumbrados a las mismas dinámicas y muchos no se interesan por la Biología, es decir que pierden interés al no tener actividades que los motiven e interesen por conocer la materia en cuestión.

- También se encontró que las profesoras no realizan prácticas de campo, ya que no poseen el conocimiento necesario para realizarlas, pero tampoco se interesan por conocer acerca de este.

- La falta de conocimiento contextual por parte de las profesoras es otro gran limitante para el desarrollo de los procesos de enseñanza de Ciencias Naturales en el Municipio, ya que si bien ellas están interesadas por los problemas ambientales(basuras, limpieza de quebradas, purificación del agua) que surgen en Floresta, están dejando de la lado la parte Paleobilógica (riqueza fosilífera), la cual es tan importante en la identificación y reconocimiento del patrimonio natural que posee el municipio. 
Memorias del VII Encuentro Nacional de Experiencias en la Enseñanza de la Biología y la Educación Ambiental y II Congreso Nacional de Investigación en la Enseñanza de la Biología

- Se encontró que las profesoras al realizar la entrevista no responden a las preguntas formuladas, además realizando la comparación de la entrevista con lo observado en las clases, las respuestas resultan incongruentes y descontextualizadas ya que varias respuestas son en una amplia medida falsas.

- Al analizar las observaciones de clases y desde la experiencia que se realiza en la práctica, se puede decir que los profesores debemos estar en constante cambio e innovación e implementación de estrategias didácticas que conlleven a una enseñanza contextualizada que nos permita a los practicantes permita tanto a nosotros como a los estudiantes explorar posibilidades diferentes de aprendizaje, las cuales no siempre tiene que llevar a seguir las indicaciones de un programa, de un libro o simplemente la utilización del tablero constantemente.

- No fu posible realizar las prácticas de campo, ya que los permisos e inconvenientes económicos y logísticos por parte de la institución impidieron la ejecución de las mismas, pero se realizaron una serie de guías de campo con las cuales la profesora encargada del área gestionará los permisos y las realizara con los estudiantes de noveno grado.

- Se puede evidenciar que las profesoras no se interesan por los temas que tienen que ver con evolución, ya que ellas articulan lo general y se quedan en ello, no pasan a lo especifico ni lo relacionan con el contexto, porque "parece ser" que las creencias religiosas son una gran barrera en la enseñanza de este tema

- A partir de la práctica se genera una reflexión acerca de la importancia de la investigación en la educación, ya que por medio de ella se pueden generar críticas importantes que conlleven a cambios en los modos de enseñanza y en la implementación de estrategias didácticas.

- Sería interesante que las guías que se dejan planteadas sería interesante que se realizaran para que las profesoras tengan en cuenta que la implementación de Prácticas de campo son importantes para el reconocimiento del contexto y patrimonio natural del Municipio por parte de los estudiantes y de ellas. Además que reconozcan a esta como una estrategia fundamental en la enseñanza de las cencías.

- Las prácticas pedagógicas integrales contribuyen a reconocer y contrastar diferentes contextos y ver cómo en estos espacios determinan en el profesor 
Bio-grafía Escritos sobre la Biología y su Enseñanza.

Edición Extra-Ordinaria. ISSN 2027-1034 P.p 658 - 665

Memorias del VII Encuentro Nacional de Experiencias en la Enseñanza de la

Biología y la Educación Ambiental y II Congreso Nacional de Investigación en la Enseñanza de La Biología

las estrategias didácticas que pueden utilizar y ayuda a determinar los modelos con los cuales el profesor en formación inicial determina su práctica.

\section{Referencias Bibliográficas}

AMÓRTEGUI, E (2011). Concepciones sobre prácticas de campo y su relación con el Conocimiento Profesional del Profesor de futuros docentes de Biología de la Universidad Pedagógica Nacional. Tesis para optar al título de Magíster en Educación. Universidad Pedagógica Nacional. Colombia, Bogotá DC

BALDAIA, Ludovina (2006). El Cambio de las Concepciones Didácticas sobre las Prácticas, en la enseñanza de la Biología. En: Alambique. Didáctica de las Ciencias Experimentales. 47. Pp. 23-29

CAMPOS, S.(2009) Práctica de Campo del Eje Curricular Organización - Un Encuentro con la Paleobiología. Bio-grafía, escritos sobre la biología y su enseñanza. 2 (3).

CERDA, H. (2001). Los elementos de la investigación: como reconocerlos, diseñarlos y construirlos. Editorial el Búho. Bogotá. Colombia.

GIL, D. (2006). Las enseñanzas prácticas en materias paleontológicas. II Encuentro sobre experiencias grupales innovadoras en la docencia universitaria. universidad complutense, Madrid, 26- 27 junio 2006.

VALBUENA, E. (2007). El Conocimiento Didáctico del Contenido Biológico. Estudio de las concepciones disciplinares y didácticas de futuros docentes de la Universidad Pedagógica Nacional (Colombia). Tesis doctoral. Universidad Complutense de Madrid. 